\title{
ON BELLMAN'S EQUATIONS WITH VMO COEFFICIENTS*
}

\author{
NICOLAI V. KRYLOV ${ }^{\dagger}$
}

Abstract. We present a result about solvability in $W_{p}^{2}, p>d$, in the whole space $\mathbb{R}^{d}$ of Bellman's equations with VMO "coefficients". Parabolic equations are touched upon as well.

Key words. Vanishing mean oscillation, fully nonlinear equations, Bellman's equations.

AMS subject classifications. 35J60

1. Main result. Let $\mathbb{R}^{d}$ be the Euclidean space of points $x=\left(x^{1}, \ldots, x^{d}\right), x^{i} \in$ $\mathbb{R}=(-\infty, \infty)$. Fix a $\delta \in(0,1)$ and denote by $\mathcal{S}_{\delta}$ the set of symmetric $d \times d$-matrices $a=\left(a^{i j}\right)$ satisfying

$$
\delta|\xi|^{2} \leq a^{i j} \xi^{i} \xi^{j} \leq \delta^{-1}|\xi|^{2}, \quad \forall \xi \in \mathbb{R}^{d} .
$$

Let $\Omega$ be a separable metric space and assume that for any $\omega \in \Omega$ and $x \in \mathbb{R}^{d}$ we are given $a(\omega, x) \in \mathcal{S}_{\delta}, b(\omega, x) \in \mathbb{R}^{d}$, and $c(\omega, x), f(\omega, x) \in \mathbb{R}$. We assume that these functions are measurable in $x$ for each $\omega$, continuous in $\omega$ for each $x$, and

$$
\begin{gathered}
|b(\omega, x)|+c(\omega, x) \leq K, \quad c(\omega, x) \geq 0, \quad \forall \omega, x, \\
\bar{f}(x):=\sup _{\omega \in \Omega}|f(\omega, x)|<\infty \quad \forall x,
\end{gathered}
$$

where $K$ is a fixed constant. Observe that, owing to the continuity of $f$ in $\omega$ and separability of $\Omega$, the function $\bar{f}$ is measurable. For $r>0$ and $x \in \mathbb{R}^{d}$ set

$$
B_{r}(x)=\left\{y \in \mathbb{R}^{d}:|x-y|<r\right\}, \quad B_{r}=B_{r}(0) .
$$

For a measurable set $\Gamma \subset \mathbb{R}^{d}$ by $|\Gamma|$ we denote its volume. In Section 4 we will use the same notation for measurable $\Gamma \subset \mathbb{R}^{d+1}$. Introduce,

$$
(u)_{\Gamma}=f_{\Gamma} u(x) d x=\frac{1}{|\Gamma|} \int_{\Gamma} u(x) d x
$$

In particular,

$$
(a)_{B_{r}(x)}(\omega)=f_{B_{r}(x)} a(\omega, y) d y
$$

In the following assumption there is a parameter $\theta \in(0,1]$, whose value will be specified later.

Assumption 1.1. There exists an $R_{0} \in(0, \infty)$ such that for any $r \in\left(0, R_{0}\right]$ and $x \in \mathbb{R}^{d}$ we have

$$
f_{B_{r}(x)} \sup _{\omega \in \Omega}\left|a(\omega, y)-(a)_{B_{r}(x)}(\omega)\right| d y \leq \theta
$$

\footnotetext{
*Received December 26, 2009; accepted for publication May 5, 2010. The work was partially supported by NSF Grant DMS-0653121.

${ }^{\dagger}$ School of Mathematics, 127 Vincent Hall, University of Minnesota, Minneapolis, MN 55455, USA (krylov@math.umn.edu).
} 
Observe that if $a$ is independent of $\omega$ (semilinear equations), and for any $\theta>0$ there is an $R_{0}>0$ such that (1.1) is satisfied for any $r \in\left(0, R_{0}\right]$ and $x \in \mathbb{R}^{d}$, then $a \in V M O$.

For constant $\lambda>0$ we will be considering the following equation

$$
\sup _{\omega \in \Omega}\left[a^{i j}(\omega, x) D_{i j} u(x)+b^{i}(\omega, x) D_{i} u(x)-(c(\omega, x)+\lambda) u(x)+f(\omega, x)\right]=0
$$

in $\mathbb{R}^{d}$. Of course, $D_{i}=\partial / \partial x^{i}, D_{i j}=D_{i} D_{j}$. By $D u$ we denote the gradient of $u$ and $D^{2} u$ the Hessian matrix of $u$. If $D$ is an open set and $p \geq 1$, by $\mathcal{L}_{p}(D)$ we denote the usual Lebesgue space and by $W_{p}^{2}(D)$ the usual Sobolev space. Denote $\mathcal{L}_{p}=\mathcal{L}_{p}\left(\mathbb{R}^{d}\right)$, $W_{p}^{2}=W_{p}^{2}\left(\mathbb{R}^{d}\right)$. We say that a function $u \in W_{p}^{2}$ satisfies (1.2) in $\mathbb{R}^{d}$ if (1.2) holds almost everywhere in $\mathbb{R}^{d}$.

The main information about the solvability of (1.2) will be obtained while studying its reduced form

$$
\sup _{\omega \in \Omega}\left[a^{i j}(\omega, x) D_{i j} u(x)+f(\omega, x)\right]=0
$$

TheOREm 1.2. Let $p>d$. Then there exists a constant $\theta \in(0,1)$ depending only on $p, d$, and $\delta$ such that, if Assumption 1.1 holds with this $\theta$, then there exists a $\lambda_{0}=\lambda_{0}(\delta, p, K, d) \geq 0$ such that for any $\lambda \geq \lambda_{0}$ and $u \in W_{p}^{2}$ satisfying (1.2) we have

$$
\lambda\|u\|_{\mathcal{L}_{p}}+\left\|D^{2} u\right\|_{\mathcal{L}_{p}} \leq N\|\bar{f}\|_{\mathcal{L}_{p}}
$$

where $N=N(\delta, p, d)$. Moreover, for any $\lambda>\lambda_{0}$ there is a unique solution of (1.2) in $W_{p}^{2}$.

Previously the $W_{p}^{2}$-estimates for a class of fully nonlinear elliptic equations were obtained by Caffarelli in [1] (see also [2]). It seems to the author that the class of equations from [2] does not include Bellman's equations like (1.3) when $\Omega$ consists of only two points:

$$
\max \left(a_{1}^{i j}(x) D_{i j} u(x)+f_{1}(x), a_{2}^{i j}(x) D_{i j} u(x)+f_{2}(x)\right)=0
$$

unless $a_{1}$ and $a_{2}$ are uniformly sufficiently close to continuous functions and $f_{1}-f_{2}$ is uniformly sufficiently close to a uniformly continuous function.

Our methods are slightly different from those from [2]. As in [2] we start from the result of [10] but then we follow more closely the general scheme from [8]. We are only dealing with equations in the whole space or interior estimates. One can probably obtain similar results for equations in half spaces and smooth domains by following the recent approach developed in [3] for linear equations and systems. In [3] one can also find an extensive list of references on linear equations with VMO coefficients.

We prove Theorem 1.2 in Section 3 after we prepare some necessary tools in the next section. In Section 4 we give some comments on how one can start obtaining similar results for parabolic equations. In the final Section 5 we prove the necessary facts from Real Analysis.

The author is sincerely grateful to Hongjie Dong for pointing out several unpleasant glitches in the first draft of the paper. 
2. Equations with constant coefficients. In this section we consider equation (1.3) under the additional assumption that $a^{i j}$ are independent of $x$, namely,

$$
\sup _{\omega \in \Omega}\left[a^{i j}(\omega) D_{i j} u(x)+f(\omega, x)\right]=0 .
$$

Lemma 2.1. Let $u \in W_{d, l o c}^{2}$. Then for any $r \in(0, \infty)$

$$
\sup _{B_{r}}\left|u(x)-x^{i}\left(u_{x^{i}}\right)_{B_{r}}-(u)_{B_{r}}\right|^{d} \leq N(d) r^{2 d} f_{B_{r}}\left|D^{2} u\right|^{d} d x .
$$

Proof. First assume that $r=1$ and set

$$
v(x)=u(x)-x^{i}\left(u_{x^{i}}\right)_{B_{1}}-(u)_{B_{1}}
$$

By Sobolev embedding theorems the left-hand side is less than a constant times

$$
\sup _{B_{1}}|v| \leq N\|v\|_{W_{d}^{2}\left(B_{1}\right)}
$$

and by Poincaré's inequality

$$
\|v\|_{W_{d}^{2}\left(B_{1}\right)}^{d}=\left\|D^{2} u\right\|_{\mathcal{L}_{d}\left(B_{1}\right)}^{d}+\left\|D u-(D u)_{B_{1}}\right\|_{\mathcal{L}_{d}\left(B_{1}\right)}^{d}+\|v\|_{\mathcal{L}_{d}\left(B_{1}\right)}^{d} \leq N\left\|D^{2} u\right\|_{\mathcal{L}_{d}\left(B_{1}\right)}^{d} .
$$

For general $r$ it suffices to use dilations. The lemma is proved.

Lemma 2.2. Let $r \in(0, \infty), \nu \geq 2$ and let $v \in C^{2}\left(\bar{B}_{\nu r}\right)$ be a solution of $(2.1)$ in $B_{\nu r}$ with $f \equiv 0$. Then there are constants $\beta \in(0,1)$ and $N$, depending only on $d$ and $\delta$, such that

$$
f_{B_{r}} f_{B_{r}}\left|D^{2} v(x)-D^{2} v(y)\right| d x d y \leq N \nu^{-2-\beta} r^{-2} \sup _{\partial B_{\nu r}}|v|
$$

Proof. Dilations show that it suffices to concentrate on $r=1 / \nu$. In that case the result follows from Theorem 5.5.8 of [7] which states that

$$
\left|D^{2} v(x)-D^{2} v(y)\right| \leq N|x-y|^{\beta} \sup _{B_{1}}|v|
$$

as long as $x, y \in B_{1 / 2}$. The lemma is proved.

Introduce $\mathbb{L}_{\delta}$ as the collection of operators $L u=a^{i j} D_{i j} u$ with $a=\left(a^{i j}\right)$ being measurable and $\mathcal{S}_{\delta}$-valued.

Lemma 2.3. Let $r \in(0, \infty)$ and let $w \in C^{2}\left(\bar{B}_{r}\right)$ be a function such that $w=0$ on $\partial B_{r}$. Then there are constants $\gamma \in(0,1]$ and $N$, depending only on $\delta$ and $d$, such that for any $L \in \mathbb{L}_{\delta}$ we have

$$
f_{B_{r}}\left|D^{2} w\right|^{\gamma} d x \leq N\left(f_{B_{r}}|L w|^{d} d x\right)^{\gamma / d}
$$

For $r=1$ the result is proved in [10] on the basis of an elliptic counterpart of Theorem 4.1. In the general case it suffices to use dilations. 
Lemma 2.4. Let $r \in(0, \infty), \nu \geq 2$, and let $u \in W_{d}^{2}\left(B_{\nu r}\right)$ be a solution of $(2.1)$ in $B_{\nu r}$. Then

$$
\begin{aligned}
& f_{B_{r}} f_{B_{r}}\left|D^{2} u(x)-D^{2} u(y)\right|^{\gamma} d x d y \\
\leq & N \nu^{d}\left(f_{B_{\nu r}} \bar{f}^{d} d x\right)^{\gamma / d}+N \nu^{-\gamma \beta}\left(f_{B_{\nu r}}\left|D^{2} u\right|^{d} d x\right)^{\gamma / d},
\end{aligned}
$$

where $N=N(\delta, d)$.

Proof. Observe that it suffices to prove the lemma for $u \in C_{b}^{\infty}\left(\bar{B}_{\nu r}\right)$. Indeed, if we denote by $u^{n}$ a sequence converging to $u$ in $W_{d}^{2}\left(B_{\nu r}\right)$, then

$$
\sup _{\omega}\left[a^{i j}(\omega, x) D_{i j} u^{n}(x)+f^{n}(\omega, x)\right]=0
$$

in $B_{\nu r}$, where $f^{n}(\omega, x)=a^{i j}(\omega, x) D_{i j}\left(u-u^{n}\right)+f(\omega, x)$, so that

$$
\left\|\sup _{\omega}\left|f^{n}(\omega, \cdot)\right|\right\|_{\mathcal{L}_{d}\left(B_{\nu r}\right)} \leq\|\bar{f}\|_{\mathcal{L}_{d}\left(B_{\nu r}\right)}+N\left\|u-u^{n}\right\|_{W_{d}^{2}\left(B_{\nu r}\right)},
$$

where the last term tends to zero as $n \rightarrow \infty$.

Next, let $v$ be a solution of (2.1) in $B_{\nu r}$ with $f^{\alpha} \equiv 0$ and the boundary condition $\hat{u}(x):=u(x)-x^{i}\left(u_{x^{i}}\right)_{B_{\nu r}}-(u)_{B_{\nu r}}$ on $\partial B_{\nu r}$. By classical results (see, for instance, [2], [4], [5], [7]) such a solution of class $C^{2}\left(\bar{B}_{\nu r}\right)$ exists. Then by Lemmas 2.1 and 2.2

$$
f_{B_{r}} f_{B_{r}}\left|D^{2} v(x)-D^{2} v(y)\right|^{\gamma} d x d y \leq N \nu^{-\gamma \beta}\left(f_{B_{\nu r}}\left|D^{2} u\right|^{d} d x\right)^{\gamma / d} .
$$

Furthermore, for $\hat{w}:=\hat{u}-v$ we have that in $B_{\nu r}$

$$
0=\sup _{\omega}\left[a^{i j}(\omega) D_{i j} \hat{w}(x)+a^{i j}(\omega) D_{i j} v(x)+f(\omega, x)\right] \leq \sup _{\omega}\left[a^{i j}(\omega) D_{i j} \hat{w}(x)+f(\omega, x)\right]
$$

and

$$
0=\sup _{\omega}\left[a^{i j}(\omega) D_{i j} \hat{w}(x)+a^{i j}(\omega) D_{i j} v(x)+f(\omega, x)\right] \geq \inf _{\omega}\left[a^{i j}(\omega) D_{i j} \hat{w}(x)+f(\omega, x)\right] .
$$

It follows that there exists an operator $L \in \mathbb{L}_{\delta}$ and a function $g$ such that $L \hat{w}+g=0$ in $B_{\nu r}$ and $|g| \leq \bar{f}$. Therefore, by Lemma 2.3 and by the fact that $D^{2} \hat{w}=D^{2} w$, where $w=u-v$, we get that

$$
\begin{aligned}
& f_{B_{r}}\left|D^{2} w\right|^{\gamma} d x \leq \nu^{d} f_{B_{\nu r}}\left|D^{2} w\right|^{\gamma} d x \leq N \nu^{d}\left(f_{B_{\nu r}} \bar{f}^{d} d x\right)^{\gamma / d}, \\
& f_{B_{r}} f_{B_{r}}\left|D^{2} w(x)-D^{2} w(y)\right|^{\gamma} d x d y \leq N \nu^{d}\left(f_{B_{\nu r}} \bar{f}^{d} d x\right)^{\gamma / d} .
\end{aligned}
$$

By combining this with (2.3) we get (2.2) and the lemma is proved.

Lemma 2.4 plays the main role in the proof of Theorem 1.2, a particular case of which is the following theorem about apriori estimates and the solvability of Bellman's equations with constant coefficients. 
Theorem 2.5. Let $p>d$. (i) Let $u \in W_{p}^{2}$ satisfy (2.1) in $\mathbb{R}^{d}$. Then there is a constant $N=N(\delta, p, d)$ such that

$$
\left\|D^{2} u\right\|_{\mathcal{L}_{p}} \leq N\|\bar{f}\|_{\mathcal{L}_{p}} .
$$

(ii) There exists a $\lambda_{0}=\lambda_{0}(\delta, p, K, d) \geq 0$ such that for any $\lambda \geq \lambda_{0}$ and $u \in W_{p}^{2}$ satisfying

$$
\sup _{\omega \in \Omega}\left[a^{i j}(\omega) D_{i j} u(x)+b^{i}(\omega, x) D_{i} u(x)-(c+\lambda)(\omega, x) u(x)+f(\omega, x)\right]=0
$$

in $\mathbb{R}^{d}$ we have

$$
\lambda\|u\|_{\mathcal{L}_{p}}+\left\|D^{2} u\right\|_{\mathcal{L}_{p}} \leq N\|\bar{f}\|_{\mathcal{L}_{p}},
$$

where $N=N(\delta, p, d)$. Moreover, for any $\lambda>\lambda_{0}$ there is a unique solution of (2.5) in $W_{p}^{2}$. Finally, if $K=0$, one can take $\lambda_{0}=0$.

Proof. (i) We use notation (5.3) for the filtration of dyadic cubes and $\mu(d x)=d x$. We also note that one can apply Lemma 2.4 after shifting the origin. Then we easily get that for any $\nu \geq 2$

$$
\left(D^{2} u\right)_{\gamma}^{\sharp} \leq N \nu^{d / \gamma} \mathbb{M}^{1 / d}\left(\bar{f}^{d}\right)+N \nu^{-\beta} \mathbb{M}^{1 / d}\left(\left|D^{2} u\right|^{d}\right)
$$

on $\mathbb{R}^{d}$, where $\mathbb{M} g$ is the classical maximal function of $g$ and $N=N(\delta, d)$.

It follows by Theorem 5.3 and the Hardy-Littlewood theorem on maximal functions that (recall that $p>d$ )

$$
\left\|D^{2} u\right\|_{\mathcal{L}_{p}} \leq N \nu^{d / \gamma}\|\bar{f}\|_{\mathcal{L}_{p}}+N \nu^{-\beta}\left\|D^{2} u\right\|_{\mathcal{L}_{p}},
$$

where $N=N(\delta, p, d)$. The arbitrariness of $\nu \geq 2$ now leads to (2.4).

(ii) By standard arguments all assertions in (ii) follow from its last one, to prove which it suffices to prove apriori estimate (2.6). Observe that by (2.4) we have (recall that $b=c=0$ )

$$
\left\|D^{2} u\right\|_{\mathcal{L}_{p}} \leq N\|\bar{f}\|_{\mathcal{L}_{p}}+N \lambda\|u\|_{\mathcal{L}_{p}},
$$

which shows that it suffices to prove that

$$
\lambda\|u\|_{\mathcal{L}_{p}} \leq N\|\bar{f}\|_{\mathcal{L}_{p}} .
$$

Of course, we may assume that $\lambda>0$ and, as in the proof of Lemma 2.4, we may assume that $u \in C_{0}^{\infty}$. Then we take an operator $L \in \mathbb{L}_{\delta}$ and a function $g$ such that $|g| \leq \bar{f}$ and $L u-\lambda u+g=0$. By Theorem 3.5.15 and Lemma 3.5.5 of [7] we have

$$
\lambda\|u\|_{\mathcal{L}_{p}} \leq N\|g\|_{\mathcal{L}_{p}} \leq N\|\bar{f}\|_{\mathcal{L}_{p}},
$$

with $N=N(\delta, d, p)$ provided that $\lambda \geq N$. Dilations show that the latter requirement can be reduced to $\lambda>0$. Actually, Theorem 3.5.15 of [7] is only proved there for $p=d$, but the proof is based on the results of Section 3.2 of [7], which treats general $p \geq d$ (see Theorem 3.2.3 there). Therefore, we can repeat the corresponding arguments in the proofs of Theorem 3.5.15 and Lemma 3.5.5 of [7] almost word for word and get what we need. A different way to obtain the first estimate in (2.9) is to refer directly to a rather old result of [6] (see Lemma 7 there), but this would require the reader to know Itô's formula from the theory of Itô integrals. This way even has an advantage because it shows that $N$ in (2.8) is independent of $p$. The theorem is proved. 
3. Proof of Theorem 1.2. We start with a few observations regarding equations with variable $a^{i j}$.

Lemma 3.1. Let $\kappa \in(1, \infty), r \in(0, \infty), \nu \geq 2$, and let $u \in W_{d}^{2}$ be a solution of (1.3) in $\mathbb{R}^{d}$. Assume that $u=0$ outside $B_{R_{0}}\left(x_{0}\right)$ for some $x_{0} \in \mathbb{R}^{d}$. Then

$$
\begin{gathered}
f_{B_{r}} f_{B_{r}}\left|D^{2} u(x)-D^{2} u(y)\right|^{\gamma} d x d y \leq N \nu^{d}\left(f_{B_{\nu r}} \bar{f}^{d} d x\right)^{\gamma / d} \\
+N \nu^{d}\left(f_{B_{\nu r}}\left|D^{2} u\right|^{\kappa d} d x\right)^{\gamma /(\kappa d)} \theta^{(1-1 / \kappa) \gamma / d}+N \nu^{-\gamma \beta}\left(f_{B_{\nu r}}\left|D^{2} u\right|^{d} d x\right)^{\gamma / d},
\end{gathered}
$$

where $N=N(\delta, d)$.

Proof. We basically repeat the proof of Lemma 6.2 .2 of [8]. Fix a $\nu \geq 2$ and an $r \in(0, \infty)$ and introduce

$$
\bar{a}^{i j}(\omega)=\left(a^{i j}\right)_{B_{R_{0}}} \quad \text { if } \quad \nu r \geq R_{0}, \quad \bar{a}^{i j}(\omega)=\left(a^{i j}\right)_{B_{\nu r}} \quad \text { if } \quad \nu r<R_{0} .
$$

Observe that

$$
\sup _{\omega \in \Omega}\left[\bar{a}^{i j}(\omega) D_{i j} u+\tilde{f}(\omega, x)\right]=0
$$

where

$$
\tilde{f}(\omega, x)=\left(a^{i j}-\bar{a}^{i j}\right)(\omega, x) D_{i j} u+f(\omega, x)
$$

Denote

$$
\tilde{a}(x)=\sup _{\omega \in \Omega}|a(\omega, x)-\bar{a}(\omega)|
$$

By Lemma 2.4

$$
\begin{gathered}
f_{B_{r}} f_{B_{r}}\left|D^{2} u(x)-D^{2} u(y)\right|^{\gamma} d x d y \leq N \nu^{d} I^{\gamma / d} \\
+N \nu^{d}\left(f_{B_{\nu r}} \bar{f}^{d} d x\right)^{\gamma / d}+N \nu^{-\gamma \beta}\left(f_{B_{\nu r}}\left|D^{2} u\right|^{d} d x\right)^{\gamma / d},
\end{gathered}
$$

where

$$
I=f_{B_{\nu r}} \tilde{a}^{d}\left|D^{2} u\right|^{d} d x=f_{B_{\nu r}} \tilde{a}^{d}\left|D^{2} u\right|^{d} I_{B_{R_{0}}} d x \leq J_{1}^{1 / \kappa} J_{2}^{1-1 / \kappa}
$$

with

$$
J_{1}=f_{B_{\nu r}}\left|D^{2} u\right|^{\kappa d} d x, \quad J_{2}=f_{B_{\nu r}} \tilde{a}^{\kappa d /(\kappa-1)} I_{B_{R_{0}}} d x \leq N f_{B_{\nu r}} \tilde{a} I_{B_{R_{0}}} d x .
$$

If $\nu r \geq R_{0}$, then

$$
J_{2} \leq N(\nu r)^{-d} \int_{B_{R_{0}}} \tilde{a} d x \leq N(\nu r)^{-d} R_{0}^{d} f_{B_{R_{0}}} \tilde{a} d x \leq N \theta
$$


In case $\nu r<R_{0}$ we have

$$
J_{2} \leq N f_{B_{\nu r}} \tilde{a} d x \leq N \theta
$$

Hence,

$$
I \leq N\left(f_{B_{\nu r}}\left|D^{2} u\right|^{\kappa d} d x\right)^{1 / \kappa} \theta^{1-1 / \kappa}
$$

and by combining this with (3.2) we come to (3.1). The lemma is proved.

Corollary 3.2. Under the assumptions of Lemma 3.1 let $p>\kappa d$. Then there is a constant $N_{1}=N_{1}(\delta, p, \kappa, d)$ such that

$$
\left\|D^{2} u\right\|_{\mathcal{L}_{p}} \leq N_{1} \nu^{d / \gamma}\|\bar{f}\|_{\mathcal{L}_{p}}+N_{1}\left(\nu^{d / \gamma} \theta^{(1-1 / \kappa) / d}+\nu^{-\beta}\right)\left\|D^{2} u\right\|_{\mathcal{L}_{p}}
$$

This is obtained in the same way as (2.7).

Corollary 3.3. Let $p>d$ and let $u \in W_{p}^{2}$ be a solution of (1.3) in $\mathbb{R}^{d}$. Assume that $u=0$ outside $B_{R_{0}}\left(x_{0}\right)$ for some $x_{0} \in \mathbb{R}^{d}$. Then there exist $\theta=\theta(p, d, \delta) \in$ $(0,1]$ and $N=N(p, d, \delta)$ such that if Assumption 1.1 is satisfied with this $\theta$, then $\left\|D^{2} u\right\|_{\mathcal{L}_{p}} \leq N\|\bar{f}\|_{\mathcal{L}_{p}}$. that

Indeed, it suffices to set $2 \kappa=1+p / d$ and choose first $\nu$ and then $\theta$ in such a way

$$
N_{1}\left(\nu^{d / \gamma} \theta^{(1-1 / \kappa) / d}+\nu^{-\beta}\right) \leq 1 / 2 .
$$

Proof of Theorem 1.2. We suppose that Assumption 1.1 holds with $\theta$ from Corollary 3.3. First assume that we are given a function $u \in W_{p}^{2}$, which satisfies (1.3) in $\mathbb{R}^{d}$.

Take a nonnegative $\zeta \in C_{0}^{\infty}$ which has support in $B_{R_{0}}$ and is such that $\zeta^{p}$ integrates to one. For the parameter $x_{0} \in \mathbb{R}^{d}$ define

$$
u_{x_{0}}(x)=u(x) \zeta\left(x-x_{0}\right)
$$

and observe that

$$
\sup _{\omega \in \Omega}\left[a^{i j}(\omega, x) D_{i j} u_{x_{0}}(x)+f_{x_{0}}(\omega, x)\right]=0
$$

where

$$
\begin{aligned}
f_{x_{0}}(\omega, x)= & f(\omega, x) \zeta\left(x-x_{0}\right)-u(x) a^{i j}(\omega, x) D_{i j} \zeta\left(x-x_{0}\right) \\
& -2 a^{i j}(\omega, x)\left(D_{i} u(x)\right) D_{j} \zeta\left(x-x_{0}\right) .
\end{aligned}
$$

By Corollary 3.3

$\left\|\zeta\left(\cdot-x_{0}\right)\left|D^{2} u\right|\right\|_{\mathcal{L}_{p}}^{p} \leq N\left\|\zeta\left(\cdot-x_{0}\right) \bar{f}\right\|_{\mathcal{L}_{p}}^{p}+\left\|\left|D \zeta\left(\cdot-x_{0}\right)\right||D u|\right\|_{\mathcal{L}_{p}}^{p}+\left\|\left|D^{2} \zeta\left(\cdot-x_{0}\right)\right| u\right\|_{\mathcal{L}_{p}}^{p}$.

Upon integrating through this estimate we get

$$
\left\|D^{2} u\right\|_{\mathcal{L}_{p}}^{p} \leq N_{1}\|\bar{f}\|_{\mathcal{L}_{p}}^{p}+N_{2}\left(\|D u\|_{\mathcal{L}_{p}}^{p}+\|u\|_{\mathcal{L}_{p}}^{p}\right)
$$


where $N_{1}=N_{1}(p, d, \delta)$ and $N_{2}=N_{2}\left(p, d, \delta, R_{0}\right)$.

If $u \in W_{p}^{2}$ is a solution of (1.2), then by absorbing the first- and zeroth-order terms into $f$ we see that

$$
\left\|D^{2} u\right\|_{\mathcal{L}_{p}} \leq N_{1}\left(\|\bar{f}\|_{\mathcal{L}_{p}}+\lambda\|u\|_{\mathcal{L}_{p}}\right)+N_{2}\left(\|D u\|_{\mathcal{L}_{p}}+\|u\|_{\mathcal{L}_{p}}\right)
$$

and if $\lambda \geq \lambda_{0}\left(p, d, \delta, R_{0}\right)$, then multiplicative inequalities yield

$$
\left\|D^{2} u\right\|_{\mathcal{L}_{p}} \leq N_{1}\left(\|\bar{f}\|_{\mathcal{L}_{p}}+\lambda\|u\|_{\mathcal{L}_{p}}\right),
$$

where $N_{1}$ still depends only on $p, d, \delta$. Applying the results of [7] or [6] as in the proof of Theorem 2.5, we obtain that

$$
\lambda\|u\|_{\mathcal{L}_{p}}+\left\|D^{2} u\right\|_{\mathcal{L}_{p}} \leq N_{1}\|\bar{f}\|_{\mathcal{L}_{p}} .
$$

After that it suffices to repeat the corresponding argument from the proof of Theorem 2.5 .

4. Comments on parabolic equations. Denote by $\mathfrak{L}$ the set of operators $L$ of the form

$$
L=\partial_{t}+a^{i j}(t, x) D_{i j}+b^{i}(t, x) D_{i}-c(t, x), \quad \partial_{t}=\frac{\partial}{\partial t},
$$

where $a(t, x)=\left(a^{i j}(t, x)\right)$ is an $\mathcal{S}_{\delta}$-valued, $b(t, x)=\left(b^{i}(t, x)\right)$ is an $\mathbb{R}^{d}$-valued, and $c(t, x)$ is a real-valued measurable functions defined on $\mathbb{R}^{d+1}=\left\{(t, x): t \in \mathbb{R}, x \in \mathbb{R}^{d}\right\}$ satisfying

$$
|b|+c \leq K, \quad c \geq 0 .
$$

Let $\mathfrak{L}_{0}$ be a subset of $\mathfrak{L}$ consisting of operators with infinitely differentiable coefficients.

For $\rho, r>0$ introduce

$$
\begin{gathered}
C_{\rho, r}=(0, \rho) \times B_{r}, \quad \partial^{\prime} C_{\rho, r}=\left([0, \rho] \times \partial B_{r}\right) \cup\left(\{\rho\} \times B_{r}\right), \\
C_{\rho, r}(t, x)=(t, x)+C_{\rho, r}, \quad \partial^{\prime} C_{\rho, r}(t, x)=(t, x)+\partial^{\prime} C_{\rho, r} .
\end{gathered}
$$

Theorem 4.1. Let $u \in W_{d+1}^{1,2}\left(C_{2,1}\right)$ and assume that $u \geq 0$ on $\partial^{\prime} C_{2,1}$ and there exists an operator $L \in \mathfrak{L}$ such that $L u \leq 0$ in $C_{2,1}$. Then there exist constants $\gamma=\gamma(\delta, d, K) \in(0,1)$ and $N=N(\delta, d, K)$ such that for any $\lambda>0$

$$
\left|C_{1,1}(1,0) \cap\{-L u \geq \lambda\}\right| \leq N \lambda^{-\gamma} u^{\gamma}(0,0) .
$$

Here is a consequence of this theorem, which can be used in constructing the theory of parabolic Bellman's equations along the lines in Sections 2 and 3.

Corollary 4.2. Let $w \in W_{d+1, l o c}^{1,2}\left(C_{2,1}\right) \cap C\left(\bar{C}_{2,1}\right)$. Then there are constants $\gamma \in(0,1]$ and $N$, depending only on $\delta, K$, and $d$, such that for any $L \in \mathfrak{L}$ we have

$$
\int_{C_{1,1}(1,0)}\left|D^{2} w\right|^{\gamma} d x d t \leq N\left(\int_{C_{2,1}}|L w|^{d+1} d x d t\right)^{\gamma /(d+1)}+N \sup _{\partial^{\prime} C_{2,1}}|w|^{\gamma} .
$$


This corollary is deduced from Theorem 4.1 in the same way as the theorem in [10] is deduced from estimate (2.1) of [10], the only difference being that instead of the elliptic Alexandrov estimate one uses the parabolic one.

To prove Theorem 4.1 we need an auxiliary construction, but first we observe that replacing $u$ with $u / \lambda$ reduces the general case to the one with $\lambda=1$.

For $q \in[0,1]$ denote by $\mathfrak{U}_{q}$ the set of functions $u$, which are bounded and continuous along with $\partial_{t} u, D u, D^{2} u$ in $\bar{C}_{2,1}$ and such that

(i) $u=0$ on $\partial^{\prime} C_{2,1}$;

(ii) there exists an operator $L \in \mathfrak{L}_{0}$ such that $L u \leq 0$ in $C_{2,1}$ and

$$
\left|C_{1,1}(1,0) \cap\{L u \leq-1\}\right| \geq q\left|C_{1,1}\right| .
$$

Finally introduce

$$
m(t, x, q)=\inf \left\{u(t, x): u \in \mathfrak{U}_{q}\right\}, \quad(t, x) \in \bar{C}_{2,1} .
$$

REMARK 4.3. Denote by $m^{\prime}(t, x, q)$ the function called $m(t, x, q)$ in Section 4.1 of [7]. Then obviously $m^{\prime}(t, x, q)=m(1+t, x, q)$. In particular, by Lemmas 4.1 .3 and 4.1.4 of [7] for any $\kappa \in(0,1)$ there exist $q_{0} \in(0,1)$ (close to 1$)$ and $m_{0}>0$, depending only on $\delta, d, K$, and $\kappa$ such that

$$
m(t, x, q) \geq m_{0}
$$

for $q \in\left[q_{0}, 1\right]$ and $(t, x) \in \bar{C}_{\kappa^{2}, \kappa}$.

REMARK 4.4. By Lemma 4.1.1 of [7] we know that for $u$ from Theorem 4.1 it holds that

$$
u(0,0) \geq m(0,0, q)
$$

if $q$ satisfies

$$
q\left|C_{1,1}\right|=\left|C_{1,1}(1,0) \cap\{L u \leq-1\}\right| .
$$

Therefore, to prove the theorem, we only need to prove that

$$
N m(0,0, q) \geq q^{1 / \gamma}
$$

for $N$ and $\gamma$ depending only on $d, \delta, K$.

We also need to use Lemma 4.1 .6 of [7]. Before stating it we introduce some notation. Let $\Gamma$ be a measurable subset of $C_{1,1}(1,0)$ and let $q, \eta, \zeta \in(0,1)$ be some numbers. Denote by $\mathfrak{B}=\mathfrak{B}(\Gamma, q)$ the collection of $Q=C_{\rho^{2}, \rho}\left(t_{0}, x_{0}\right)$ such that $Q \subset C_{1,1}(1,0)$ and

$$
|Q \cap \Gamma| \geq q\left|C_{1,1}\right|
$$

If $Q=C_{\rho^{2}, \rho}\left(t_{0}, x_{0}\right) \in \mathfrak{B}$ we set

$$
Q^{\prime}=\left(t_{0}, x_{0}\right)-C_{\eta^{-1} \rho^{2}, \rho}, \quad Q^{\prime \prime}=\left(t_{0}-\eta^{-1} \rho^{2}, x_{0}\right)+C_{\eta^{-1} \rho^{2} \zeta^{2}, \rho \zeta} .
$$

Imagine that the $t$-axis is pointed up vertically. Then $Q^{\prime}$ is immediately adjacent to $Q$ from below, the two cylinders have a common base, and along the $t$-axis $Q^{\prime}$ is 
$\eta^{-1}$ times longer than $Q$. It is quite possible that part of $Q^{\prime}$ comes out of $C_{1,1}(1,0)$ or, for that matter, out of $C_{2,1}$. The cylinder $Q^{\prime \prime}$ is obtained from $Q^{\prime}$ by parabolic compression centered at the center of the lower base of $Q^{\prime}$, the compression coefficient being $\zeta^{-1}$. Finally, denote

$$
\Gamma^{\prime \prime}=\bigcup_{Q \in \mathfrak{B}} Q^{\prime \prime}
$$

Here is Lemma 4.1.6 of [7].

Lemma 4.5. If $|\Gamma| \leq q\left|C_{1,1}\right|$, then

$$
\left|\Gamma^{\prime \prime}\right| \geq\left(1-(1-q) 3^{-d-1}\right)^{-1}(1+\eta)^{-1} \zeta^{d+2}|\Gamma| .
$$

Proof of Theorem 4.1. We are going to slightly modify the proof of Theorem 4.1.2 of [7] while concentrating on (4.2). Fix some $\eta, \zeta \in(0,1)$ to be specified later and such that

$$
\zeta^{2} \leq 1-2 \eta
$$

Next, fix a $\kappa \in(0,1)$ such that $\kappa^{2} \geq 1 / 2$ and set

$$
\mu(q)=\inf \{m(0, x, q):|x| \leq \kappa\} .
$$

By Remark 4.3 there is a $q_{0} \in(0,1)$ and $m_{0}>0$ such that

$$
\mu\left(q_{0}\right) \geq m_{0} .
$$

Next, we take some $0<q^{\prime}<q^{\prime \prime}<1$ and try to relate $\mu\left(q^{\prime}\right)$ to $\mu\left(q^{\prime \prime}\right)$. To this end take a $u \in \mathfrak{U}_{q^{\prime}}$ and let

$$
\Gamma=C_{1,1}(1,0) \cap\{L u \leq-1\}
$$

where $L$ is the operator associated with $u$. From chosen $\Gamma, q_{0}, \eta$, and $\zeta$ we construct the set $\Gamma^{\prime \prime}$ as before Lemma 4.5 by taking there $q_{0}$ in place of $q$ and consider two cases:

(i) $\left|\Gamma^{\prime \prime} \backslash C_{1,1}(1,0)\right| \leq\left(q^{\prime \prime}-q^{\prime}\right)\left|C_{1,1}\right|$,

(ii) $\left|\Gamma^{\prime \prime} \backslash C_{1,1}(1,0)\right|>\left(q^{\prime \prime}-q^{\prime}\right)\left|C_{1,1}\right|$.

Case (i). Denote by $\tilde{u}$ and $\tilde{v}$ the $W_{d+1}^{1,2}\left(C_{2,1}\right)$-solutions of

$$
L \tilde{u}=-I_{\Gamma}, \quad L \tilde{v}=-I_{\Gamma_{0}}
$$

vanishing on $\partial^{\prime} C_{2,1}$, where $\Gamma_{0}=\Gamma^{\prime \prime} \cap C_{1,1}(1,0)$. Since the coeficients of $L$ are infinitely differentiable, such solutions exist. There are two possibilities: either

(a) $|\Gamma| \geq q_{0}\left|C_{1,1}\right|$,

or

(b) $|\Gamma|<q_{0}\left|C_{1,1}\right|$.

Under condition (a) by definition

$$
u(0, x) \geq \mu\left(q_{0}\right), \quad|x| \leq \kappa .
$$

In case (b) by definition and Lemma 4.5

$$
q^{\prime}\left|C_{1,1}\right| \leq|\Gamma| \leq\left(1-\left(1-q_{0}\right) 3^{-d-1}\right)(1+\eta) \zeta^{-d-2}\left|\Gamma^{\prime \prime}\right| .
$$


Moreover, by assumption

$$
\left|\Gamma^{\prime \prime}\right|=\left|\Gamma^{\prime \prime} \backslash C_{1,1}(1,0)\right|+\left|\Gamma_{0}\right| \leq\left(q^{\prime \prime}-q^{\prime}\right)\left|C_{1,1}\right|+\left|\Gamma_{0}\right| .
$$

It follows that

$$
\left|\Gamma_{0}\right| \geq q^{\prime \prime}\left|C_{1,1}\right|
$$

if

$$
(1+\xi) q^{\prime} \geq 2 q^{\prime \prime}
$$

where

$$
\xi:=\left(1-\left(1-q_{0}\right) 3^{-d-1}\right)^{-1}(1+\eta)^{-1} \zeta^{d+2} .
$$

Obviously there exist $\eta=\eta\left(q_{0}\right) \in(0,1)$ and $\zeta=\zeta\left(q_{0}\right) \in(0,1)$ such that (4.3) is satisfied and $\xi=\xi\left(q_{0}\right)>1$, so that (4.5) holds for some $q^{\prime}<q^{\prime \prime}$. Since $q_{0}$ depends only on $\delta, K, d$, and $\kappa$, so do $\eta, \zeta$, and $\xi$. We fix such $\eta$ and $\zeta$ from this moment on. Then by definition

$$
\tilde{v}(0, x) \geq \mu\left(q^{\prime \prime}\right), \quad|x| \leq \kappa .
$$

We thus have estimated $\tilde{v}$ from below in case (i), (b) for $q^{\prime}<q^{\prime \prime}$ satisfying (4.5). By the maximum principle $u \geq \tilde{u}$ and to estimate $u$ from below it suffices to estimate $\tilde{u}$ from below in terms of $\tilde{v}$. This will be done by use of Lemma 4.1.5 of [7].

If $\left(t_{0}, x_{0}\right) \in \Gamma_{0}$, then there exists a cylinder $Q \in \mathfrak{B}$ such that $\left(t_{0}, x_{0}\right) \in Q^{\prime \prime}$. Define $\left(t_{1}, x_{1}\right)$ and $\tau, \rho$ from the equation

$$
C_{\tau, \rho}\left(t_{1}, x_{1}\right)=Q_{1}:=\left(Q^{\prime} \cup Q\right) \cap C_{2,1},
$$

so that

$$
Q=C_{\rho^{2}, \rho}\left(\tau+t_{1}-\rho^{2}, x_{1}\right) .
$$

Furthermore, owing to (4.3), the distance from $\left(t_{0}, x_{0}\right)$ to the bottom of $Q$ is bigger than

$$
\eta^{-1} \rho^{2}-\eta^{-1} \rho^{2} \zeta^{2} \geq 2 \rho^{2}
$$

In particular,

$$
1<t_{0} \leq\left(\tau+t_{1}-\rho^{2}\right)-2 \rho^{2} .
$$

Next let $\bar{u}$ and $\bar{v}$ be the $W_{d+1}^{1,2}\left(Q_{1}\right)$-solutions of

$$
L \bar{u}=-N_{0} I_{\Gamma}, \quad L \bar{v}=-I_{\Gamma_{0}},
$$

vanishing on $\partial^{\prime} Q_{1}$, where the constant $N_{0}$ will be specified later in such a way that

$$
\bar{u}\left(t_{0}, x_{0}\right) \geq \bar{v}\left(t_{0}, x_{0}\right) .
$$

If we can do this, then by Lemma 4.1.5 of [7] we have $N_{0} \tilde{u} \geq \tilde{v}$ on $\bar{C}_{2,1}$ and for $q^{\prime}<q^{\prime \prime}$ satisfying (4.5)

$$
u(0, x) \geq \tilde{u}(0, x) \geq N_{0}^{-1} \tilde{v}(0, x) \geq N_{0}^{-1} \mu\left(q^{\prime \prime}\right), \quad|x| \leq \kappa .
$$


To prove (4.7), observe that by the maximum principle $\bar{v}(t, x) \leq t_{1}+\tau-t$, so that

$$
\bar{v}\left(t_{0}, x_{0}\right) \leq\left(1+\eta^{-1}\right) \rho^{2} .
$$

On the other hand, by the choice of $Q$ we have $q_{0}|Q| \leq|\Gamma \cap Q|$. This inequality is preserved under the parabolic dilation $x \rightarrow \rho^{-1} x, t \rightarrow \rho^{-2} t$ which transforms $\bar{u}$ into a function $\hat{u}$, which satisfies $\hat{L} \hat{u}=-N_{0} \rho^{2} I_{\hat{\Gamma}}$ with an $\hat{L} \in \mathfrak{L}_{0}$ and $\hat{\Gamma}$ being the image of $\Gamma$. Observe that the hyperplane $t=\tau+t_{1}-2 \rho^{2}$ passes at a distance $\rho^{2}$ from $Q$ and it intersects $Q_{1}$ above $t=t_{0}$. Hence, by definition

$$
\bar{u}\left(\tau+t_{1}-2 \rho^{2}, x\right) \geq N_{0} \rho^{2} \mu\left(q_{0}\right), \quad\left|x-x_{1}\right| \leq \kappa \rho .
$$

Since $\left|x_{0}-x_{1}\right| \leq(1-\zeta) \rho$ and the distance between the hyperplanes $t=\tau+t_{1}-2 \rho^{2}$ and $t=t_{0}$ is bigger than $\rho^{2}$ and less than $\eta^{-1} \rho^{2}$, it follows by Lemma 4.1.3 of [7] that

$$
\bar{u}\left(t_{0}, x_{0}\right) \geq \alpha N_{0} \rho^{2} \mu\left(q_{0}\right),
$$

where $\alpha>0$ depends only on $d, \delta, K$, and $\kappa$. By taking

$$
N_{0}=\alpha^{-1}\left(1+\eta^{-1}\right) \mu^{-1}\left(q_{0}\right)
$$

and recalling (4.9) we come to (4.7) and thus (4.8) is established in case (i), (b) for $q^{\prime}<q^{\prime \prime}$ satisfying (4.5), so that generally in case (i) (recall (4.4)) for those $q^{\prime}, q^{\prime \prime}$

$$
u(0, x) \geq \min \left(\mu\left(q_{0}\right), N_{0}^{-1} \mu\left(q^{\prime \prime}\right)\right), \quad|x| \leq \kappa .
$$

The arbitrariness in the choice of $u$ implies that

$$
\mu\left(q^{\prime}\right) \geq \min \left(\mu\left(q_{0}\right), N_{0}^{-1} \mu\left(q^{\prime \prime}\right)\right),
$$

which after introducing

$$
\hat{\mu}(q)=\min \left(\mu\left(q_{0}\right), \mu(q)\right)
$$

yields

$$
\left.\hat{\mu}\left(q^{\prime}\right) \geq N_{0}^{-1} \hat{\mu}\left(q^{\prime \prime}\right)\right) \quad \text { if } \quad(1+\xi) q^{\prime} \geq 2 q^{\prime \prime} .
$$

Case (ii). First we claim that for some $(t, x) \in \Gamma^{\prime \prime}$ it holds that $t<q^{\prime}-q^{\prime \prime}+1$. Indeed, otherwise $\Gamma^{\prime \prime} \subset C_{q^{\prime \prime}-q^{\prime}, 1}(1,0)$ and $\left|\Gamma^{\prime \prime}\right| \leq\left(q^{\prime \prime}-q^{\prime}\right)\left|C_{1,1}\right|$. It follows that there is a cylinder

$$
Q=C_{\rho^{2}, \rho}\left(t_{0}, x_{0}\right) \in \mathfrak{B}
$$

such that $Q^{\prime}$ contains points in the half-space $t<q^{\prime}-q^{\prime \prime}+1$. Since $q^{\prime}<q^{\prime \prime}, q^{\prime}-q^{\prime \prime}+1<$ 1 and $Q^{\prime}$ is adjacent to $Q \subset C_{1,1}(1,0)$, this implies that the height of $Q^{\prime}$ is at least $q^{\prime \prime}-q^{\prime}$, that is,

$$
\rho^{2} \eta^{-1} \geq q^{\prime \prime}-q^{\prime}, \quad \rho^{2} \geq \eta\left(q^{\prime \prime}-q^{\prime}\right)
$$

Moreover, by definition $|\Gamma \cap Q| \geq q_{0}|Q|$ and by using dilations and the maximum principle we see that

$$
u\left(t_{0}-\rho^{2}, x\right) \geq \mu\left(q_{0}\right) \rho^{2}, \quad\left|x-x_{0}\right| \leq \kappa \rho .
$$


If $t_{0}-\rho^{2} \geq 1 / 4$, then (4.12) by Lemma 4.1 .3 of [7] implies that

$$
u(0, x) \geq \mu\left(q_{0}\right) \varepsilon \rho^{n}, \quad|x| \leq \kappa,
$$

where $\varepsilon>0$ and $n \geq 1$ depend only on $\delta, K, d$, and $\kappa$. In particular (see (4.11)),

$$
u(0, x) \geq \mu\left(q_{0}\right) \varepsilon \eta^{n}\left(q^{\prime \prime}-q^{\prime}\right)^{n}, \quad|x| \leq \kappa .
$$

On the other hand, by Remark 4.3 for $t_{0}-\rho^{2} \leq t \leq t_{0}-\rho^{2}+\kappa^{2} \rho^{2}$ and $\left|x-x_{0}\right| \leq \kappa \rho$ it holds that

$$
u(t, x) \geq m_{0} \rho^{2} .
$$

In addition, if $t_{0}-\rho^{2}<1 / 4$, then in the above inequality one can take $t=\kappa^{2} / 2$ since $t_{0} \geq 1, \rho^{2}>3 / 4, \rho^{2} \leq 1$ (also recall that $\kappa^{2} \geq 1 / 2$ ) and

$$
t_{0}-\rho^{2}<1 / 4 \leq \kappa^{2} / 2 \leq t_{0}-\rho^{2}+\kappa^{2} / 2 \leq t_{0}-\rho^{2}+\kappa^{2} \rho^{2} .
$$

Hence, $u\left(\kappa^{2} / 2, x\right) \geq m_{0} \rho^{2}$ for $\left|x-x_{0}\right| \leq \kappa \rho$ and as above

$$
u(0, x) \geq m_{0} \varepsilon \eta^{n}\left(q^{\prime \prime}-q^{\prime}\right)^{n}, \quad|x| \leq \kappa,
$$

with $\varepsilon$ and $n$ of the same kind as in (4.13) or just the same if we choose the minimum of $\varepsilon$ 's and the maximum of $n$ 's. Again the arbitrariness of $u$ yields $\mu\left(q^{\prime}\right) \geq m_{0} \varepsilon \eta^{n}\left(q^{\prime \prime}-\right.$ $\left.q^{\prime}\right)^{n}$, which after reducing $\varepsilon$ if necessary, so that $\mu\left(q_{0}\right) \geq m_{0} \varepsilon \eta^{n}$ leads to

$$
\hat{\mu}\left(q^{\prime}\right) \geq m_{0} \varepsilon \eta^{n}\left(q^{\prime \prime}-q^{\prime}\right)^{n} .
$$

As a result of considering the two cases (i) and (ii) we get that there exist $\varepsilon_{0} \in$ $(0,1)$ and $n_{0} \geq 1$ depending only on $\delta, K, d$, and $\kappa$, such that for any $0<q^{\prime}<q^{\prime \prime}<1$ such that $(1+\xi) q^{\prime} \geq 2 q^{\prime \prime}$ we have

$$
\hat{\mu}\left(q^{\prime}\right) \geq \varepsilon_{0} \min \left(\left(q^{\prime \prime}-q^{\prime}\right)^{n_{0}}, \hat{\mu}\left(q^{\prime \prime}\right)\right) .
$$

We also know that $\hat{\mu}(q) \geq m_{0}>0$ for $q \geq q_{0}$.

We may certainly assume that $\varepsilon_{0} \leq \bar{\varepsilon}:=2 /(1+\xi)$ (recall that $\xi>1$ ) and we claim that for $q_{k}=\bar{\varepsilon}^{k} q_{0}, k=0,1,2, \ldots$, we have

$$
\hat{\mu}\left(q_{k}\right) \geq \varepsilon_{0}^{k n_{0}} \chi, \quad \chi:=\min \left(\mu\left(q_{0}\right), q_{0}^{n_{0}}(1-\bar{\varepsilon})^{n_{0}}\right) .
$$

To prove the claim we use induction. If $k=0,(4.15)$ is obvious. If it is true for a $k$, then $q_{k}-q_{k+1}=\bar{\varepsilon}^{k} q_{0}(1-\bar{\varepsilon})$

$$
\left(q_{k}-q_{k+1}\right)^{n_{0}}=\bar{\varepsilon}^{k n_{0}} q_{0}^{n_{0}}(1-\bar{\varepsilon})^{n_{0}} \geq \varepsilon_{0}^{k n_{0}} \chi,
$$

so that by (4.14) and the fact that $(1+\xi) q_{k+1}=2 q_{k}$

$$
\hat{\mu}\left(q_{k+1}\right) \geq \varepsilon_{0} \min \left(\varepsilon_{0}^{k n_{0}} \chi, \hat{\mu}\left(q_{k}\right)\right) \geq \varepsilon_{0} \varepsilon_{0}^{k n_{0}} \chi \geq \varepsilon_{0}^{(k+1) n_{0}} \chi .
$$

This proves (4.15) and shows that, if we define $r>1$ so that $\varepsilon_{0}^{n_{0}}=\bar{\varepsilon}^{r}$, then $\hat{\mu}\left(q_{k}\right) \geq N q_{k}^{r}$ with $r, N>0$ depending only on $\delta, K, d$, and $\kappa$. By observing that $\hat{\mu}$ is an increasing function we obtain that $\hat{\mu}(q) \geq N q^{r}, \mu(q) \geq N q^{r}$ for $q \leq 1$. Finally, since $m(0,0, q) \geq \mu(q)$ if in the construction of $\mu$, we take any $\kappa>0$, say $\kappa^{2}=1 / 2$, we come to (4.2) with $\gamma=1 / r$, which, as it is explained in Remark 4.4, proves the theorem. 
5. Appendix. We will be working in the setting of Chapter 3 of [8] using notation different from the previous sections of the present article. Thus, $(\Omega, \mathcal{F}, \mu)$ is a measurable space with $\sigma$-finite measure $\mu$ satisfying $\mu(\Omega)=\infty$. For $\Gamma \in \mathcal{F}$ we use the notation $|\Gamma|=\mu(\Gamma)$ and

$$
f_{\Gamma}=f_{\Gamma} f \mu(d x)=\frac{1}{|\Gamma|} \int_{\Gamma} f \mu(d x) .
$$

Next we take a filtration $\left\{\mathbb{C}_{n}: n \in \mathbb{Z}\right\}$ of partitions of $\Omega$ as in Section 3.1 of [8] and recall that for any $n \in \mathbb{Z}$ and $C \in \mathbb{C}_{n}$ there exists a unique "parent" $C^{\prime} \in \mathbb{C}_{n-1}$ such that $C \subset C^{\prime}$. It is assumed that whenever $C$ and $C^{\prime}$ are related in the above described manner we have $\left|C^{\prime}\right| \leq N_{0}|C|$, where $N_{0}$ is a constant independent of $n, C$, and $C^{\prime}$.

For functions $g$, for which it makes sense, denote

$$
g_{\mid n}(x)=f_{C_{n}(x)} g(y) \mu(d y)
$$

where $C_{n}(x)$ is the element of the family $\mathbb{C}_{n}$ containing $x$. Also

$$
\mathcal{M} g(x):=\sup _{n<\infty}|g|_{\mid n}(x) .
$$

The most relevant filtration of partitions in this paper is the dyadic cube filtration of partitions of $\mathbb{R}^{d}$ with Lebesgue measure when

$$
\begin{gathered}
\mathbb{C}_{n}=\left\{C_{n}\left(i_{1}, \ldots, i_{d}\right), i_{1}, \ldots, i_{d} \in \mathbb{Z}\right\}, \\
C_{n}\left(i_{1}, \ldots, i_{d}\right)=\left[i_{1} 2^{-n},\left(i_{1}+1\right) 2^{-n}\right) \times \ldots \times\left[i_{d} 2^{-n},\left(i_{d}+1\right) 2^{-n}\right) .
\end{gathered}
$$

In the remaining part of the section we consider two functions $u, v \in \mathcal{L}_{1}(\Omega)$ and a nonnegative measurable function $g$ on $\Omega$. Below by $I_{\mathcal{M} v(x)>\alpha \lambda}$ we mean the indicator function of the set $\{x: \mathcal{M} v(x)>\alpha \lambda\}$.

The most relevant case of Lemma 5.1 for the purposes of the present article is when $u^{C}=|u|$. In the form it is stated and for $\gamma=1$ the lemma was used in [9] while treating linear elliptic equations with rather rough coefficients.

Lemma 5.1. Let $\gamma \in(0,1]$. Assume that $|u| \leq v$ and for any $n \in \mathbb{Z}$ and $C \in \mathbb{C}_{n}$ there exists a measurable function $u^{C}$ given on $C$ such that $|u| \leq u^{C} \leq v$ on $C$ and

$$
f_{C} f_{C}\left|u^{C}(x)-u^{C}(y)\right|^{\gamma} \mu(d x) \mu(d y) \leq f_{C} g^{\gamma}(x) \mu(d x) .
$$

Then for any $\lambda>0$ we have

$$
|\{x:|u(x)| \geq \lambda\}| \leq \nu^{-1} \lambda^{-\gamma} \int_{\Omega} g^{\gamma}(x) I_{\mathcal{M} v(x)>\alpha \lambda} \mu(d x),
$$

where $\alpha=\left(2 N_{0}\right)^{-1}$ and $\nu=1-2^{-\gamma}$.

Proof. Obviously we may assume that $u \geq 0$. Fix a $\lambda>0$ and define

$$
\tau(x)=\inf \left\{n \in \mathbb{Z}: v_{\mid n}(x)>\alpha \lambda\right\} .
$$


We know that $\tau$ is a stopping time and if $\tau(x)<\infty$, then

$$
v_{\mid n}(x) \leq \lambda / 2, \quad \forall n \leq \tau(x) .
$$

We also know that $v_{\mid n} \rightarrow v \geq u$ (a.e.) as $n \rightarrow \infty$. It follows that (a.e.)

$$
\begin{gathered}
\{x: u(x) \geq \lambda\}=\{x: u(x) \geq \lambda, \tau(x)<\infty\} \\
=\left\{x: u(x) \geq \lambda, v_{\mid \tau}(x) \leq \lambda / 2\right\}=\bigcup_{n \in \mathbb{Z}} \bigcup_{C \in \mathbb{C}_{n}^{\tau}} A_{n}(C),
\end{gathered}
$$

where

$$
A_{n}(C):=\left\{x \in C: u(x) \geq \lambda, v_{\mid n}(x) \leq \lambda / 2\right\},
$$

and $\mathbb{C}_{n}^{\tau}$ is the family of disjoint elements of $\mathbb{C}_{n}$ such that

$$
\{x: \tau(x)=n\}=\bigcup_{C \in \mathbb{C}_{n}^{\tau}} C .
$$

Next, for each $n \in \mathbb{Z}$ and $C \in \mathbb{C}_{n}$ on the set $A_{n}(C)$, if it is not empty, we have $v_{\mid n}=v_{C}$ and on $A_{n}(C)$

$$
u^{\gamma}-\left(v_{C}\right)^{\gamma} \geq \lambda^{\gamma}\left(1-2^{-\gamma}\right)=\nu \lambda^{\gamma}
$$

We use this and the inequality $|a-b|^{\gamma} \geq|a|^{\gamma}-|b|^{\gamma}$ and conclude that for $x \in A_{n}(C)$

$$
\begin{gathered}
f_{C}\left|u^{C}(x)-u^{C}(y)\right|^{\gamma} \mu(d y) \geq\left(u^{C}(x)\right)^{\gamma}-f_{C}\left(u^{C}(y)\right)^{\gamma} \mu(d y) \\
\geq u^{\gamma}(x)-f_{C} v^{\gamma}(y) \mu(d y) \geq u^{\gamma}(x)-\left(v_{C}(x)\right)^{\gamma} \geq \nu \lambda^{\gamma},
\end{gathered}
$$

so that by Chebyshev's inequality

$$
\left|A_{n}(C)\right| \leq \nu^{-1} \lambda^{-\gamma} \int_{C} f_{C}\left|u^{C}(x)-u^{C}(y)\right|^{\gamma} \mu(d y) \mu(d x) .
$$

It follows by assumption (5.1) that

$$
\begin{gathered}
\left|A_{n}(C)\right| \leq \nu^{-1} \lambda^{-\gamma} \int_{C} g^{\gamma} \mu(d x), \\
|\{x: u(x) \geq \lambda\}| \leq \nu^{-1} \lambda^{-\gamma} \sum_{n \in \mathbb{Z}} \sum_{C \in \mathbb{C}_{n}^{\tau}} \int_{C} g^{\gamma} \mu(d x)=\nu^{-1} \lambda^{-\gamma} \int_{\Omega} g^{\gamma} I_{\tau<\infty} \mu(d x) .
\end{gathered}
$$

It only remains to observe that $\{\tau<\infty\}=\{\mathcal{M} v>\alpha \lambda\}$. The lemma is proved.

COROLlary 5.2. Under the assumptions of Lemma 5.1 for any $p>\gamma$ we have

$$
\int_{\Omega}|u|^{p} \mu(d x) \leq \beta\left(\int_{\Omega}(\mathcal{M} v)^{p} \mu(d x)\right)^{1-\gamma / p}\left(\int_{\Omega} g^{p} \mu(d x)\right)^{\gamma / p}
$$


where $\beta=\nu^{-1}(1-\gamma / p)^{-1} \alpha^{\gamma-p}$.

Indeed,

$$
\begin{gathered}
\int_{\Omega}|u|^{p} \mu(d x)=\int_{0}^{\infty}\left|\left\{x:|u(x)| \geq \lambda^{1 / p}\right\}\right| d \lambda \\
\leq \nu^{-1} \int_{\Omega} g^{\gamma} \int_{0}^{\infty} \lambda^{-\gamma / p} I_{\mathcal{M} v>\alpha \lambda^{1 / p}} d \lambda \mu(d x)=\beta \int_{\Omega} g^{\gamma}(\mathcal{M} v)^{p-\gamma} \mu(d x)
\end{gathered}
$$

and it only remains to use Hölder's inequality.

The second statement of the following theorem for $\gamma=1$ is, actually, the Fefferman-Stein theorem.

Theorem 5.3. For $\gamma \in(0,1]$ define

$$
u_{\gamma}^{\sharp}(x)=\sup _{n} \sup _{C \in \mathbb{C}_{n}: x \in C}\left(f_{C} f_{C}|u(z)-u(y)|^{\gamma} \mu(d z) \mu(d y)\right)^{1 / \gamma} .
$$

Then for $p>\gamma$

$$
\int_{\Omega}|u|^{p} \mu(d x) \leq \beta\left(\int_{\Omega}(\mathcal{M} u)^{p} \mu(d x)\right)^{1-\gamma / p}\left(\int_{\Omega}\left(u_{\gamma}^{\sharp}\right)^{p} \mu(d x)\right)^{\gamma / p} .
$$

In particular, by Hardy-Littlewood theorem, for $p>1$ and $u \in \mathcal{L}_{p}$ we have

$$
\|u\|_{\mathcal{L}_{p}} \leq N\left\|u_{\gamma}^{\sharp}\right\|_{\mathcal{L}_{p}},
$$

where $N=\beta^{1 / \gamma} q^{(p-\gamma) / \gamma}, q=p /(p-1)$.

This is a simple consequence of Corollary 5.2 since, obviously, for $n \in \mathbb{Z}, C \in \mathbb{C}_{n}$, and $v=u^{C}=|u|$ we have $|u| \leq u^{C} \leq v$, and for any $x \in C$

$$
f_{C} f_{C}\left|u^{C}(z)-u^{C}(y)\right|^{\gamma} \mu(d z) \mu(d y) \leq f_{C} f_{C}|u(z)-u(y)|^{\gamma} \mu(d z) \mu(d y) \leq\left(u_{\gamma}^{\sharp}(x)\right)^{\gamma},
$$

so that

$$
f_{C} f_{C}\left|u^{C}(z)-u^{C}(y)\right|^{\gamma} \mu(d z) \mu(d y) \leq f_{C}\left(u_{\gamma}^{\sharp}\right)^{\gamma} \mu(d x) .
$$

\section{REFERENCES}

[1] L. A. CAFFARELLI, Interior a priori estimates for solutions of fully nonlinear equations, Annals of Mathematics, 130 (1989), pp. 189-213.

[2] L. A. Caffarelli and X. Cabré, Fully nonlinear elliptic equations, Colloq. Publ., vol. 43, Amer. Math. Soc., Providence, RI, 1995.

[3] Honguie Dong and Doyoon Kim, On the $L_{p}$-solvability of higher order parabolic and elliptic systems with BMO coefficients, Preprint 2009.

[4] L. C. Evans, Partial differential equations, Amer. Math. Soc., Providence, RI, 1998.

[5] D. Gilbarg and N. S. Trudinger, Elliptic partial differential equations of second order, Reprint of the 1998 edition, Classics in Mathematics, Springer-Verlag, Berlin, 2001.

[6] N. V. KRYlov, Control of a solution of a stochastic integral equation, Teoriya Veroyatnostei i eye Primeneniya, 17:1 (1972), pp. 111-128 (Russian); English translation in Theor. Probability Appl., 17:1 (1972), pp. 114-131. 
[7] N. V. KRYLOv, Nonlinear elliptic and parabolic equations of second order, Nauka, Moscow, 1985 (Russian); English translation: Reidel, Dordrecht, 1987.

[8] N. V. KRYLov, Lectures on elliptic and parabolic equations in Sobolev spaces, Amer. Math. Soc., Providence, RI, 2008.

[9] N. V. KRYLOV, Second-order elliptic equations with variably partially VMO coefficients, Journal of Functional Analysis, 257 (2009), pp. 1695-1712.

[10] Fang-Hua Lin, Second derivative $L^{p}$-estimates for elliptic equations of nondivergent type, Proc. Amer. Math. Soc., 96:3 (1986), pp. 447-451. 
